\title{
Detection of spin correlations in optical lattices by light scattering
}

\author{
Inés de Vega, J. Ignacio Cirac, and D. Porras \\ Max-Planck-Institut für Quantenoptik, Hans-Kopfermann-Strasse 1, Garching D-85748, Germany
}

(Received 5 October 2007; published 29 May 2008)

\begin{abstract}
We show that spin correlations of atoms in an optical lattice can be reconstructed by coupling the system to the light, and by measuring correlations between the emitted photons. This principle is the basis for a method to characterize states in quantum computation and simulation with optical lattices. As examples, we study the detection of spin correlations in a quantum magnetic phase, and the characterization of cluster states.
\end{abstract}

DOI: 10.1103/PhysRevA.77.051804

PACS number(s): 42.50.Ct, 03.75.Hh, 03.75.Lm, 03.75.Mn

Ultracold atoms in optical lattices open exciting prospects for the investigation of quantum many-body phases in a highly controllable setup. For example, spin-spin interactions between atoms in a Mott insulator state can be tuned in such a way that one can simulate a rich variety of models from quantum magnetism [1]. Furthermore, this system is ideally suited to implement a quantum register for quantum computation [2], and to realize multiparticle entangled states with cold controlled collisions $[3,4]$. An important benchmark in this direction has been the creation of a cluster state [5], since it can be used as a resource for measurement-based universal quantum computation [6,7].

A major disadvantage of this setup is that atoms are separated by optical wavelengths, and thus it is difficult to address them individually. This prevents the direct measurement of the spatial dependence of spin-spin correlations, which is essential in the characterization of quantum phases. For these reasons, the development of accurate methods to measure properties of atomic operators is basic to the usefulness of optical lattices for quantum computation and simulation. A possible solution relies on the detection of the atom number distribution in time-of-flight (TOF) imaging. Within this technique, atomic density operators in momentum space are measured by taking absorption images of the expanding cloud, after having switched off the trapping potentials. Also, density-density correlation functions in momentum space [8-10] are reconstructed by detecting statistical correlations between different images. On the other hand, one may use quantum nondemolition techniques on the atom-light interaction in order to determine certain atomic collective observables $[11,12]$.

In this Rapid Communication, we describe an alternative approach that allows one to measure spin correlations without switching off the optical potential. Furthermore, it is not necessary to drive the atoms out of the strongly correlated regime, thus avoiding certain complications that are met in TOF measurements of interacting fermions. Our method is based on off-resonant scattering of an incident laser with trapped atoms. Scattered photons with a certain polarization are coupled to certain spin operators. In that way, correlations between photons emitted in different directions are proportional to ground-state correlations of spin operators in momentum space. Different detection schemes, such as photon counting or homodyne detection, may give information on different types of correlations. One of the strengths of the method is that it is based on photon detection, and not on atom detection. For that reason, detection of any possible spin-spin correlation is achieved here naturally, just by controlling the polarization of the lasers and the polarization of the detected photons. The correlation of an arbitrary number of spin operators can be achieved by considering correlations of different detections.

We consider an optical lattice filled with $N$ atoms in a Mott insulator state with at most one atom per site. Each atom carries an arbitrary ground-state hyperfine spin $J$, and has an optical dipole transition to an excited-state manifold of spin $J^{\prime}$. This transition is coupled with an off-resonant laser of frequency $\omega_{L}$, wave vector $\mathbf{k}_{L}$, and a certain linear polarization $\sigma_{L}$. Photons are then scattered with momentum $\mathbf{k}$ and two different polarizations $\sigma$, such that $\sigma \neq \sigma_{L}$. Light modes with momentum $\mathbf{k}$ are coupled to atomic operators $J_{\sigma}^{\Delta \mathbf{k}}=(1 / \sqrt{N}) \sum_{j} J_{\sigma}^{j} e^{i \Delta \mathbf{k} \cdot \mathbf{r}_{j}}$, with $\Delta \mathbf{k}=\mathbf{k}-\mathbf{k}_{L}$, where $J_{\sigma}^{j}$ are atomic spin operators, and the vector $\mathbf{r}_{j}$ denotes the position of the atoms in the lattice. Notice that $J_{\sigma}^{\Delta \mathbf{k}}$ has a periodicity $2 \pi / r_{0}$, where $r_{0}$ is the lattice constant, which gives rise to Bragg peaks in the emission spectra. We present the following results. (i) Measuring correlations between photons with momenta $\mathbf{k}$, we reconstruct correlations between atomic spin operators $J_{\sigma}^{\Delta \mathbf{k}}$, such that $k=k_{\mathrm{L}}$. Full access to correlations both in momentum and position space requires that $k_{L} r_{0}$ $>\sqrt{2} \pi(\sqrt{3} \pi)$ in two-dimensional (2D) (3D) square lattices. However, these conditions are not always necessary to characterize a state in the momentum space. (ii) Measured correlations are in the ground state provided that the measurement time $T \ll 1 / \Gamma$, where $\Gamma$ is the spontaneous emission rate. The validity of this approximation is studied by simulating the emission properties of interacting atoms in a lattice by means of a bosonic description of spin excitations. (iii) As an illustration, we discuss the measurement of a magnetic phase, which is a relevant example to quantum simulation, as well as a measurement of a cluster state in a 2D lattice, which has important applications in quantum computation.

The incident laser has a large detuning $\Delta=\omega_{J^{\prime}}-\omega_{L}$, with respect to the uppermost level of $J^{\prime}$, with frequency $\omega_{J^{\prime}}$. In that situation, the excited manifold can be adiabatically eliminated $[13,14]$, and the evolution of any operator $A$ acting on the spin $J$ manifold can be written in terms of an effective Hamiltonian for light-matter interaction $(d / d t) A$ $=(i / \hbar)\left[H_{\mathrm{lm}}^{\mathrm{eff}}, A\right]$,

$$
H_{\mathrm{lm}}^{\mathrm{eff}}=\sum_{j}\left(\mathbf{E}_{\mathrm{tot}}^{j \dagger} \stackrel{\leftrightarrow}{\alpha_{j}} \mathbf{E}_{\mathrm{tot}}^{j}+\text { H.c. }\right)
$$

The electromagnetic field is usually expressed in terms of the two orthonormal polarization vectors $\hat{\mathbf{e}}_{\mathbf{k} l} \perp \mathbf{k}(l=1,2)$ and the 
corresponding creation (annihilation) operators $a_{\mathbf{k} l}^{\dagger}\left(a_{\mathbf{k} l}\right)$. Nevertheless, here it is more convenient to express the field appearing in Eq. (1) in the laboratory frame, so that $\mathbf{E}_{\text {tot }}^{j}$ $=\Sigma_{\sigma}\left(\widetilde{E}_{\sigma}^{j-} \hat{\sigma}+\hat{E}_{\sigma}^{j+} \hat{\sigma}+E_{L}^{j+} \hat{\sigma}_{L}\right)$ with $\sigma_{L}, \sigma=x, y, z$. In the last definition, $\tilde{E}_{\sigma}^{j+}=\Sigma_{\mathbf{k}} \epsilon_{\mathbf{k}} a_{\mathbf{k} \sigma} e^{-i \Delta_{k}^{+} t+i \mathbf{k} \cdot \mathbf{r}_{j}}$ is a fast rotating field [15], $\hat{E}_{\sigma}^{j+}=\sum_{\mathbf{k}} \epsilon_{\mathbf{k}} a_{\mathbf{k} \sigma} e^{-i \Delta_{k}^{t+i \mathbf{k} \cdot \mathbf{r}_{j}}}$ is a slowly rotating field, and $E_{L}^{j+}$ $=\epsilon_{L} e^{-i \mathbf{k}_{L} \cdot \mathbf{r}_{j}}$ is the incident laser. Also, $\widetilde{E}_{\sigma}^{j-}=\left(\widetilde{E}_{\sigma}^{j+}\right)^{\dagger}, \hat{E}_{\sigma}^{j-}$ $=\left(\hat{E}_{\sigma}^{j+}\right)^{\dagger}$, and the constants are $\epsilon_{k}=\sqrt{\hbar \omega_{k} /\left(2 \epsilon_{0} v\right)}$ and $\Delta_{k}^{ \pm}$ $=\omega_{k} \pm \omega_{L}$. The photon operators have been defined in the laboratory frame, $\quad a_{\mathbf{k} x}=a_{\mathbf{k} 1} \cos \theta \cos \phi+a_{\mathbf{k} 2} \sin \phi, \quad a_{\mathbf{k} y}$ $=a_{\mathbf{k} 1} \cos \theta \sin \phi-a_{\mathbf{k} 2} \cos \phi$, and $a_{\mathbf{k} z}=-a_{\mathbf{k} 1} \sin \theta$, with $\theta$ and $\phi$ the angular coordinates of the wave vector $\mathbf{k}$ in the laboratory frame.

The polarizability appearing in Eq. (1) is written as $\overleftrightarrow{\alpha}_{j}$ $=d^{2} / \hbar \Delta\left[a_{0}(\Delta) \mathbf{I}_{j}+i a_{1}(\Delta) \mathbf{J}_{j} \times+a_{2}(\Delta) \stackrel{\leftrightarrow}{T}\right]$, where $\stackrel{\leftrightarrow}{T}_{j}$ is a tensorial component that depends on squares of the spin operators and $\mathbf{I}_{j}$ is a diagonal matrix of spin operator identities $[13,14]$. The coefficients $a_{l}(\Delta)$ with $(l=0,1,2)$ depend on the particular transition and tend to an asymptotic value for large $\Delta$. We consider transitions for which at large $\Delta, \quad a_{2}(\Delta) \rightarrow 0$ $[11,13,14]$, e.g., the cesium atom $D_{2}$ line at $J=4 \rightarrow J^{\prime}$ $=3,4,5$. The term proportional to $a_{0}$ does not describe a coupling between photons and atomic spins, and contributes to the emitted field with $\sigma_{L}$-polarized photons. These photons can be distinguished from the ones that are coupled to atomic spin operators, which come from the term proportional to $a_{1}$ and are mostly emitted with a different polarization $\sigma \neq \sigma_{L}$. Hence, for measuring the atomic spin, only the term proportional to $a_{1}$ shall be taken into account in $H_{\text {eff. }}$. Let us consider, for example, a laser with polarization $\sigma_{L}=z$. In that case,

$$
H^{\mathrm{eff}}=i g \sum_{j}\left[J_{x}^{j}\left(E_{y}^{j-}-E_{y}^{j+}\right)-J_{y}^{j}\left(E_{x}^{j-}-E_{x}^{j+}\right)\right]
$$

where $g=\epsilon_{L} d^{2} a_{1} / \Delta$ and $E_{\sigma}^{j-}=\widetilde{E}_{\sigma}^{j-} e^{-i \mathbf{k}_{L} \cdot \mathbf{r}_{j}}-\hat{E}_{\sigma}^{j-} e^{i \mathbf{k}_{L} \cdot \mathbf{r}_{j}}$ for $\sigma$ $=x, y$. Here, we have neglected the so-called reabsorption terms, which correspond to processes in which a spontaneously emitted photon is reabsorbed, and considered only the dominant terms that are proportional to $\epsilon_{L}$, which correspond to processes in which a laser photon is involved. The Hamiltonian (2) describes a coupling between the $y$-polarized $\left(x\right.$-polarized) emitted photons and the spin operators $J_{x}^{j}\left(J_{y}^{j}\right)$. Different laser polarizations give rise to the scattering of photons that are coupled to other spin operators.

We show first how the light-matter coupling (2) allows us to obtain spin correlations of the kind $\left\langle J_{\alpha}^{\Delta \mathbf{k}} J_{\beta}^{-\Delta \mathbf{k}}\right\rangle$, by detecting $N_{\alpha \beta}^{\hat{\mathbf{k}}}(T)=\left[v /(2 \pi)^{3}\right] \int d k k^{2}\left\langle a_{\mathbf{k} \alpha}^{\dagger}(T) a_{\mathbf{k} \beta}(T)\right\rangle$, where $v$ is the quantization volume. The diagonal elements $N_{\alpha \alpha}^{\hat{\mathbf{k}}}(T)$ are the number of photons emitted in the direction $\hat{\mathbf{k}}$ during a time $T$. The nondiagonal terms are obtained by combining photon number measurements of circularly and linearly polarized photons within the $x-y$ plane. From the Heisenberg equations of the field operators, we find

$$
\begin{aligned}
N_{\alpha \beta}^{\hat{\mathbf{k}}}(T)= & N\left(\frac{L}{2 \pi}\right)^{3} \int d k k^{2}\left(g \epsilon_{k} \epsilon_{L}\right)^{2} \sum_{\alpha^{\prime} \beta^{\prime}} \mathcal{M}_{\alpha \alpha^{\prime}}^{\mathbf{k}} \mathcal{M}_{\beta \beta^{\prime}}^{\mathbf{k}} \\
& \times \int_{-T}^{T} d \tau \int_{0}^{T} d t e^{-i \Delta_{\mathbf{k}} \tau}\left\langle J_{\alpha^{\prime}}^{\Delta \mathbf{k}}(t+\tau / 2) J_{\beta^{\prime}}^{-\Delta \mathbf{k}}(t-\tau / 2)\right\rangle
\end{aligned}
$$

where the sum goes over $\alpha^{\prime}, \beta^{\prime}=x, y, z$, and we have defined $\mathcal{M}_{\alpha \alpha^{\prime}}^{\mathbf{k}}=\Sigma_{\beta} \epsilon^{\sigma_{L} \alpha \alpha^{\prime}}\left[a_{\mathbf{k} \alpha^{\prime}}, a_{\mathbf{k} \beta}^{\dagger}\right]$, where $\epsilon^{\sigma_{L} \alpha \alpha^{\prime}}$ is the Levi-Cività symbol. We now consider some approximations in order to simplify Eq. (3). First, the evolution of the atomic correlation due to the atomic Hamiltonian $H_{\text {at }}$ occurs in a time scale $T_{A}=1 / \epsilon$ (where $\epsilon$ is a typical eigenenergy of $H_{\mathrm{at}}$ ), much larger than the light-matter interaction processes considered, and can therefore be neglected. Second, $T$ can be made short enough to ensure that the dependency of the correlation on $\tau$ can be neglected. Finally, assuming $T \gg \tau_{C}$ (where $\tau_{C}$ is the environmental decay time) allows us to extend to infinity the integration limits of the first integral. Hence, we can write

$$
N_{\alpha \beta}^{\hat{\mathbf{k}}}(T) \approx N \Gamma_{0} \sum_{\alpha^{\prime} \beta^{\prime}} \mathcal{M}_{\alpha \alpha^{\prime}}^{\mathbf{k}} \mathcal{M}_{\beta \beta^{\prime}}^{\mathbf{k}} \int_{0}^{T} d t\left\langle J_{\alpha^{\prime}}^{\Delta \mathbf{k}}(t) J_{\beta^{\prime}}^{-\Delta \mathbf{k}}(t)\right\rangle,
$$

where the constant $\Gamma_{0}=g^{2}\left(\frac{L}{2 \pi}\right)^{3} \pi \epsilon_{L} \int d k k^{2} \epsilon_{k}^{2} \delta\left(k-k_{L}\right)$ is the spontaneous-emission rate of a single atom. Moreover, if $T$ $\ll 1 / \Gamma$, where $1 / \Gamma$ is an estimate of the spontaneousemission decaying time of the system that may be renormalized by collective effects, we can write

$$
\tilde{N}_{\alpha \beta}^{\hat{\mathbf{k}}}(T) \approx N \Gamma_{0} \sum_{\alpha^{\prime} \beta^{\prime}} \mathcal{M}_{\alpha \alpha^{\prime}}^{\mathbf{k}} \mathcal{M}_{\beta \beta^{\prime}}^{\mathbf{k}} T\left\langle J_{\alpha^{\prime}}^{\Delta \mathbf{k}}(0) J_{\beta^{\prime}}^{-\Delta \mathbf{k}}(0)\right\rangle .
$$

The validity of this approximation will be studied later in more detail, since the measuring time $T$ also has to be long enough to ensure that a sufficient number of photons to characterize the state is detected [16].

Apart from the photon number, other operators such as the field quadratures are linked to spin observables. Let us consider detection in the far-field limit, so that the detector is placed at a position $\mathbf{R}$ such that $k_{L} R \gg 1$. In that case, it can be shown that the modes that contribute more to the field are those with wave vector in directions $\hat{\mathbf{k}}=\hat{\mathbf{R}}$, with $\hat{\mathbf{R}}$ a unit vector in the direction of the detector [17]. In addition, due to energy conservation, the largest contribution to the emitted field comes from $k=k_{L}$. Hence, the positive component of the emitted field corresponding to the $\alpha$ polarization can be written as $E_{\alpha}^{+}(\mathbf{R}, T) \approx\left(\sqrt{N} \Gamma_{0} / g\right) e^{-i \omega_{L} t+i k_{L} R} \sum_{\beta} M_{\beta \alpha}^{\hat{\mathbf{R}}} J_{\beta}^{-\Delta \mathbf{R}}(0)$, where $\Delta \mathbf{R}=k_{L} \hat{\mathbf{R}}-\mathbf{k}_{L}$. Here we have followed similar approximations as in the derivation of Eq. (4), and also that $J_{\beta}^{\Delta \mathbf{k}}(\tau)$ $\approx J_{\beta}^{\Delta \mathbf{k}}(0)$ for $\tau \epsilon[0, T]$, provided again that $T \ll 1 / \Gamma$. For instance, a homodyne detection of the quadrature $\mathcal{X}_{\alpha}^{\mathbf{k}}=\epsilon_{\mathbf{k}}\left(a_{\mathbf{k} \alpha}^{\dagger}\right.$ $\left.+a_{\mathbf{k} \alpha}\right)$ is related to the spin operators in the following way:

$$
\mathcal{X}_{\alpha}^{\mathbf{k}}(T)=2 \sqrt{N} \frac{\Gamma_{0}}{g} \sum_{\beta} \mathcal{M}_{\alpha \beta}^{\mathbf{k}} J_{C \beta}^{\Delta \mathbf{k}}(0)
$$

where $\quad \mathbf{k}=k_{L} \hat{\mathbf{R}}, \quad \beta=x, y, z, \quad$ and $\quad J_{C \beta}^{\Delta \mathbf{k}}(0)$ $=\left[J_{\beta}^{\Delta \mathbf{k}}(0)+J_{\beta}^{-\Delta \mathbf{k}}(0)\right] / 2=\sum_{j} \cos \left(\Delta \mathbf{k} \cdot \mathbf{r}_{j}\right) J_{\beta}^{j} / \sqrt{N}$. From Eq. (6), 
we see that a certain quadrature is proportional to a combination of system spin operators. In order to detect only a certain spin component, e.g., $J_{C x}^{\Delta \mathbf{k}}$, the detector should be fixed $\theta$ and $\phi$ such that only $\mathcal{M}_{\alpha x} \neq 0$. Different values of $\Delta \mathbf{k}$ are scanned by changing $\hat{\mathbf{k}}_{L}$, which provides us with information of atomic spins (and their correlations) within a 2D slice in momentum space. Spin operators in the 3D momentum space may be obtained with measurements in which both the detector and the laser are moved. In that case, to extract information of a particular spin correlation requires us to invert the matrix $\mathcal{M}^{\mathbf{k}}=\left\{\mathcal{M}_{\alpha, \beta}^{\mathbf{k}}\right\}$, which in general can be done except for some particular values of $\theta$ and $\phi$ such that $\operatorname{det}[\mathcal{M}]=0$.

We turn to study further the approximation $N_{\alpha, \beta}(T)$ $\approx \tilde{N}_{\alpha, \beta}(T)$, which allows us to reconstruct ground-state correlations from the emitted photons. This approximation is valid as long as the spin coherence is not destroyed by the incident field. The idea is to calculate how much of the information that we get from the atoms corresponds to their ground state, so that the relative error

$$
\left(E_{R}^{\alpha}\right)^{2}=\frac{\int d \cos (\theta)\left(\mathcal{N}_{\alpha \alpha}^{\Omega_{\theta, \phi}}-\tilde{N}_{\alpha \alpha}^{\Omega_{\theta, \phi}}\right)^{2}}{\int d \cos (\theta)\left(\mathcal{N}_{\alpha,}^{\Omega_{\theta, \phi}}\right)^{2}}
$$

is small up to a time $T$ such that a sufficient number of photons has been emitted to obtain information of the atomic correlation. The radiative emission of an ensemble of interacting atoms poses a complicated problem, which shows the interplay between quantum dissipation and many-body effects. We address it here by considering that atoms have a well-defined magnetic ordering along the $z$ axis, such that $\left[J_{x}^{j}, J_{y}^{l}\right]=i \delta_{j, l}$, in a Holstein-Primakoff (HP) approximation. Within the HP approximation, Hamiltonian (2) yields the following system of evolution equations:

$$
\begin{gathered}
\frac{\partial\left\langle J_{x}^{\mathbf{q}}\right\rangle}{\partial t}=-U \int d \Omega_{\mathbf{k}} \delta_{1 / L}^{\mathbf{q}-\Delta \mathbf{k}}\left[M_{x y}^{\mathbf{k}}\left\langle J_{y}^{\Delta \mathbf{k}}\right\rangle+M_{x x}^{\mathbf{k}}\left\langle J_{x}^{\Delta \mathbf{k}}\right\rangle\right], \\
\frac{\partial\left\langle J_{y}^{\mathbf{q}}\right\rangle}{\partial t}=U \int d \Omega_{\mathbf{k}} \delta_{1 / L}^{\mathbf{q}+\Delta \mathbf{k}}\left[M_{y y}^{\mathbf{k}}\left\langle J_{y}^{-\Delta \mathbf{k}}\right\rangle+M_{y x}^{\mathbf{k}}\left\langle J_{x}^{-\Delta \mathbf{k}}\right\rangle\right],
\end{gathered}
$$

where $U=4 g^{2} \Gamma_{0}$, and the spin dynamics induced by $H_{\text {at }}$ during the radiative time scale has been neglected. $\delta_{1 / L}^{\mathbf{q}}$ $=\sum_{j} e^{i \mathbf{q} \cdot \mathbf{r}_{j}}$ is a function centered at $\mathbf{q}=\mathbf{0}$ with width $1 / L$. In the limit $1 / L \ll d_{0}$, spin operators vary slower than $\delta_{1 / L}^{\mathrm{q}}$ in momentum space, such that one can approximate $\int d \Omega_{\mathbf{k}} \mathcal{M}_{\alpha \beta}^{\mathbf{k}} \delta_{1 / L}^{\mathbf{q}+\mathbf{k}}\left\langle J_{\beta}^{ \pm \Delta \mathbf{k}}\right\rangle \approx\left\langle J_{\beta}^{ \pm \mathbf{q}}\right\rangle \int d \Omega_{\mathbf{k}} \mathcal{M}_{\alpha \beta}^{\mathbf{k}} \delta_{1 / L}^{\mathbf{q} \mp \Delta \mathbf{k}}$, and Eq. (8) becomes a closed system for each atomic operator with momentum q. From the quantum regression theorem, Eq. (8) can be used to evolve two operator averages and calculate the emission pattern by means of Eq. (4).

As a particular example, we consider spin-1/2 atoms, with $\left.H_{\mathrm{at}}=B \Sigma_{j} J_{z}^{j}+J \sum_{\langle i j\rangle}\right\rangle_{x}^{i} J_{x}^{j}$, where $B$ is an external magnetic field, $\langle i j\rangle$ refers to nearest neighbors, and the interaction is ferromagnetic interaction $(J<0)$. In the regime $B>|J|$, and far from the critical point, the system is in a paramagnetic phase with spins aligned along the $\hat{z}$ axis, so that

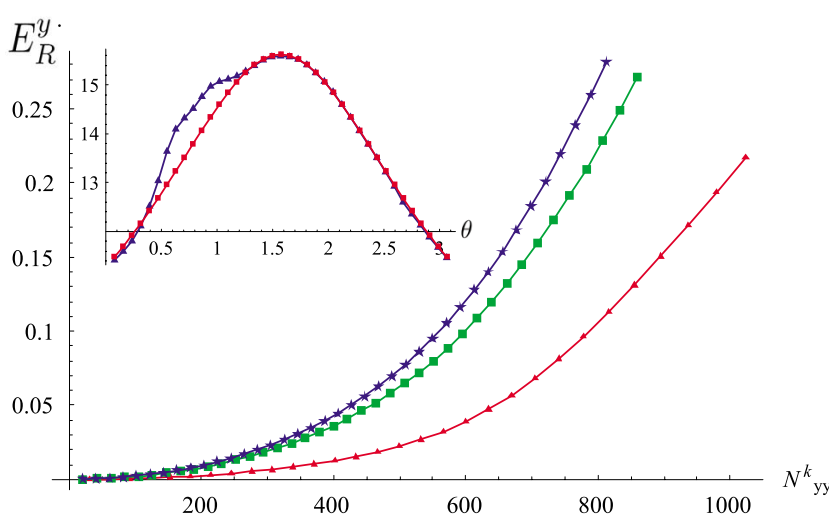

FIG. 1. (Color online) Relative error between $\mathcal{N}_{y y}^{\Omega_{\theta, \phi=0}}$ and $\tilde{N}_{y y}^{\Omega_{\theta, \phi=0}}(T)$, with respect to the number of emitted photons with $\alpha$ $=y$, integrating all over $\theta$ for $\phi=0$. Triangles, $J / B=-0.5$; boxes, $J / B=-0.1$; stars, $J / B=-0.01$. Inset: Number of $y$-polarized photons for each value of $\theta$ with $\phi=0, T=0.001$ (units of $\Gamma_{0}=1$ ), and $J / B$ $=-0.5$. Triangles, $\quad \mathcal{N}_{y y}^{\Omega_{\theta, \phi=0}}=\Gamma_{0} N \int_{0}^{T} d t\left\langle J_{x}^{\theta, \phi=0}(t) J_{x}^{\theta, \phi=0}(t)\right\rangle$ (emitted photons). Squares, $\tilde{N}_{y y}^{\Omega_{\theta, \phi=0}}(T)=\Gamma_{0} N T\left\langle J_{x}^{\theta, \phi=0}(0) J_{x}^{\theta, \phi=0}(0)\right\rangle$ (emitted photons that correspond to the ground state).

the HP approximation yields an adequate description of the quantum fluctuations in the ground state. In this approximation, the problem can be rewritten in momentum space, $H_{\mathrm{at}}=\Sigma_{\mathbf{q}}\left(2 B+J_{\mathbf{q}}\right) J_{x}^{\mathbf{q}} J_{x}^{-\mathbf{q}}+J_{y}^{\mathbf{q}} J_{y}^{-\mathbf{q}}$, where we have used the Fourier transformed spin-spin interaction, $J_{\mathbf{q}}$ $=2 J\left[\cos \left(q_{x} r_{0}\right)+\cos \left(q_{y} r_{0}\right)+\cos \left(q_{z} r_{0}\right)\right]$. Note that the description of the many-body problem in terms of spin waves suits our purpose perfectly, since it also allows us to describe the emission process. The ground-state correlations are $\left\langle J_{y}^{\mathbf{q}} J_{y}^{-\mathbf{q}}\right\rangle$ $=\sqrt{1+J_{\mathbf{q}} / 4 B}, \quad\left\langle J_{x}^{\mathbf{q}} J_{x}^{-\mathbf{q}}\right\rangle=\frac{1}{2} \sqrt{1+J_{\mathbf{q}} / 4 B}, \quad\left\langle J_{x}^{\mathbf{q}} J_{y}^{-\mathbf{q}}\right\rangle=i / 2, \quad$ and $\left\langle J_{y}^{\mathbf{q}} J_{x}^{-\mathbf{q}}\right\rangle=-i / 2$, and shall be used as initial conditions to the set of equations (8). In the inset of Fig. 1, the quantity $\mathcal{N}_{y y}^{\Omega_{\theta, \phi=0}}$ is shown for a certain small $T$ such that most of the photons come from the system ground state. In addition, the relative error (7), for $\alpha=y$ and $\phi=0$, is plotted in Fig. 1 with respect to the number of emitted photons (proportional to $T$ ). It can be seen that the error remains relatively small when enough photons (of the order of 600) have been emitted.

By measuring in different directions, it is possible to reconstruct atomic correlations in the position space. To study the conditions required for this task, we focus on a $2 \mathrm{D}$ optical lattice, which is a relevant case to the characterization of cluster states and experiments that simulate high- $T_{c}$ superconductors. We also consider that the incident laser propagates along the $z$ direction, and that the lattice is within the $x-y$ plane. Following Eq. (6), a homodyne detection of the quadrature $\mathcal{X}_{\alpha}^{\mathbf{k}}$ allows one to measure the operator $J_{C \beta}^{\Delta \mathbf{k}}$, where now we have that $\Delta \mathbf{k}=k_{\mathrm{L}}(\sin \theta \cos \phi, \sin \theta \sin \phi, \cos \theta-1)$. Then the spin operator $J_{C \beta}^{(n, m)}=J_{\beta}^{(n, m)}+J_{\beta}^{(-n,-m)}$ in the position space, with $(n, m)$ denoting a particular site within the lattice, can be obtained as an inverse Fourier transform of $J_{C \beta}^{\Delta \mathbf{k}}$,

$$
J_{C \beta}^{(n, m)}=C \int_{-1}^{1} d \tilde{k}_{x} \int_{-1}^{1} d \tilde{k}_{y} \cos \left(\tilde{k}_{x} n \pi+\tilde{k}_{y} m \pi\right) J_{C \beta}^{\Delta \mathbf{k}},
$$

where we have defined $C=\sqrt{N} / 2$, and the integration variables as $\tilde{\mathbf{k}}=\left(r_{0} / \pi\right) \Delta \mathbf{k}$. By changing the detection angles 
$(\theta, \phi)$, it is possible to measure the quantity $J_{C \beta}^{\Delta \mathbf{k}}$ for values of $\Delta \mathbf{k}$ that lie within a circle of radius $R_{0}=2 k_{\mathrm{L}} r_{0} / \pi$. The integral (9) has to be sampled with a set of values of $J_{C \beta}^{\Delta \mathbf{k}}$ within the region of integration defined by the square $\tilde{k}_{x} \in[-1,1]$ and $\tilde{k}_{y} \in[-1,1]$. Therefore, a basic requirement to obtain $J_{C \beta}^{(n, m)}$ is to choose $r_{0} k_{L} \geq \sqrt{2} \pi$, so that the integration region is contained within the circle $R_{0}$. Note that this implies that $k_{L} / k_{\text {latt }} \geq \sqrt{2}$, where $k_{\text {latt }}$ is the wavelength of the standing wave lasers. An additional homodyne detection of the quadrature $\mathcal{P}_{\alpha}^{\mathbf{k}}=i \epsilon_{\mathbf{k}}\left(a_{\mathbf{k} \alpha}^{\dagger}-a_{\mathbf{k} \alpha}\right)$ allows one to obtain the quantity $J_{S \beta}^{(n, m)}=J_{\beta}^{(n, m)}-J_{\beta}^{(-n,-m)}$, which combined with Eq. (9) can be used to measure the operator $J_{\beta}^{(n, m)}$. Following the same argument as before, the condition to get spatial correlations in $3 \mathrm{D}$ is $r_{0} k_{L} \geq \sqrt{3} \pi$. Once the $J_{\beta}^{(n, m)}$ are obtained as the inverse Fourier transforms of field quadratures, it is straightforward to calculate their correlations, which are very useful to characterize interesting states, such as cluster states $|\phi\rangle_{\mathrm{cl}}$. The latter are defined by a set of eigenvalue equations for the operator $K^{j}=\sigma_{x}^{j} \otimes_{l \in \mathcal{N}(j)} \sigma_{z}^{l}$, such that $K^{j}|\phi\rangle_{\mathrm{cl}}= \pm|\phi\rangle_{\mathrm{cl}}[6,7]$, where $\mathcal{N}(j)$ specifies the sites of all atoms $l$ that interact with an atom $j$. Hence, a cluster state in a 2D lattice can be characterized by checking that the correlation $\left\langle J_{z}^{\left(n_{1}, m_{1}\right)} J_{z}^{\left(n_{1}, m_{2}\right)} J_{x}^{\left(n_{3}, m_{3}\right)} J_{z}^{\left(n_{4}, m_{4}\right)} J_{z}^{\left(n_{5}, m_{5}\right)}\right\rangle$ is equal to \pm 1 when the operators $J_{z}$ are next neighbors of $J_{x}$. These spatial correlations can be obtained measuring $\left\langle J_{z}^{\Delta \mathbf{k}_{1}} J_{z}^{\Delta \mathbf{k}_{2}} J_{x}^{\Delta \mathbf{k}_{3}} J_{z}^{\Delta \mathbf{k}_{4}} J_{z}^{\Delta \mathbf{k}_{5}}\right\rangle$ by using a $y$-polarized laser and correlations of different homodyne detections of quadratures. Then, the inverse transform can be made following the basic relation (9) and an analogous relation for $J_{S \beta}^{(n, m)}$. This scheme can be used to measure many operator averages that characterize magnetic quantum phases, as, for example, the valence bond strength, $\left\langle\mathbf{J}_{j} \cdot \mathbf{J}_{l}\right\rangle$, where $j, l$ are nearest neighbors.

Limitations in the detection efficiency $\eta_{\text {eff }}$ can be overcome by considering a large number of experimental runs $M$, such that the total number of detected photons $N_{\text {tot }}$ $\approx \eta_{\text {eff }} \Gamma T N M$. Moreover, $M$ has to be such that the quantity $1 / \sqrt{M}$ that characterizes the statistical error for the measurement is small. An interesting point is that in contrast to TOF, there are no limitations regarding the laser shot noise: laser fluctuations can be easily distinguished, since the polarization of the emitted photons $\sigma \neq \sigma_{L}$.

We have shown how to detect spin correlations of an atom lattice within a Mott insulator state without switching off the potential. The detection is based on the fact that spin correlations in momentum are proportional to correlations of the photons that are emitted in an off-resonant scattering process. Using different photon detection techniques allows us to measure different types of spin correlations that are useful to characterize certain many-body states such as magnetic phases. A complete sampling of these correlations in momentum can be used to obtain spatial correlations, which are useful to characterize some other phases such as cluster states.

We thank Miguel Aguado for helpful discussions. Work supported by EU projects (SCALA, COVAQIAL, and CONQUEST), and Cluster of Excellence Munich-Centre for Advanced Photonics (MAP). I.D.V. acknowledges support from Ministerio de Educación y Ciencia.
[1] L.-M. Duan, E. Demler, and M. D. Lukin, Phys. Rev. Lett. 91, 090402 (2003).

[2] I. H. Deutsch and P. S. Jessen, Phys. Rev. A 57, 1972 (1998).

[3] G. K. Brennen, C. M. Caves, P. S. Jessen, and I. H. Deutsch, Phys. Rev. Lett. 82, 1060 (1999).

[4] D. Jaksch, H. J. Briegel, J. I. Cirac, C. W. Gardiner, and P. Zoller, Phys. Rev. Lett. 82, 1975 (1999).

[5] O. Mandel, M. Greiner, A. Widera, T. Rom, T. W. Hänsch, and T. Bloch, Nature (London) 425, 937 (2003).

[6] R. Raussendorf and H. J. Briegel, Phys. Rev. Lett., 86, 5188 (2001).

[7] R. Raussendorf, D. E. Browne, and H. J. Briegel, Phys. Rev. A 68, 022312 (2003).

[8] E. Altman, E. Demler, and M. D. Lukin, Phys. Rev. A 70, 013603 (2004).

[9] S. Fölling, F. Gerbier, A. Widera, O. Mandel, T. Gericke, and
T. Bloch, Nature (London) 434, 481 (2005).

[10] M. Greiner, C. A. Regal, J. T. Stewart, and D. S. Jin, Phys. Rev. Lett. 94, 110401 (2005).

[11] K. Eckert, L. Zawitkowski, A. Sanpera, M. Lewenstein, and E. S. Polzik, Phys. Rev. Lett. 98, 100404 (2007).

[12] K. Eckert, O. Romero-Isart, M. Rodriguez, M. Lewenstein, E. S. Polzik, and A. Sanpera, Nat. Phys. 4, 50 (2008).

[13] K. Hammerer, Ph.D. thesis, Max Planck Institute for Quantum Optics, Garching, Germany (unpublished).

[14] B. Julsgaard, Ph.D. thesis, University of Aarhus, Denmark (unpublished).

[15] The fast rotating term shall be included here to describe correctly the dipole-dipole interactions [17].

[16] D. F. Walls and G. J. Milburn, Quantum Optics (SpringerVerlag, Berlin, 1994).

[17] R. H. Lehmberg, Phys. Rev. A 2, 883 (1970). 